\title{
Risk factors for postoperative cerebrospinal fluid leakage after transsphenoidal surgery for pituitary adenoma: a meta-analysis and systematic review
}

Zhihuan Zhou ${ }^{1,2+}$, Feifei Zuo ${ }^{1,2+}$, Xiaoqun Chen ${ }^{1,2}$, Qinqin Zhao ${ }^{1,2}$, Mengna Luo ${ }^{1,3}$, Xiaobing Jiang ${ }^{1,2}$ and Yuyu Duan ${ }^{1,2^{*}}$

\begin{abstract}
Objective: Postoperative cerebrospinal fluid (CSF) leakage represents a challenge even for experienced pituitary surgeons. We aimed to quantitatively synthesize data from studies regarding the risk factors for postoperative CSF leakage after transsphenoidal surgery (TSS) for pituitary adenoma (PA).

Methods: PubMed, Web of Science, The Cochrane Library, Embase, China National Knowledge Infrastructure (CNKI), Wanfang database, and VIP database were searched for case-control and cohort studies, focusing on the risk factors associated with postoperative CSF leakage after TSS for PA. Pooled odds ratios (ORs) and 95\% confidence intervals were calculated to determine the risk factors.

Results: A total of 34 case-control and cohort studies involving a total of 9,144 patients with PA were included in this systematic review. The overall rate of postoperative CSF leakage after TSS for PA was 5.6\%. Tumor size, adenoma consistency, revision surgery, and intraoperative CSF leakage were independent risk factors for postoperative CSF leakage (ORs, 3.18-6.33). By contrast, the endoscopic approach showed a slight protective benefit compared with the microscopic approach in TSS (OR, 0.69).

Conclusions: This review provides a comprehensive overview of the quality of the evidence base, informing clinical staff of the importance of screening risk factors for postoperative CSF leakage after TSS for PA. More attention should be paid to PA patients at high risk for CSF leakage after TSS to reduce complications and improve prognosis.
\end{abstract}

Keywords: Cerebrospinal fluid leakage, Transsphenoidal surgery, Pituitary adenoma, Meta-analysis, Systematic review

\section{Introduction}

Pituitary adenomas (PAs) are benign neoplasms that represent the most common type of pituitary disorder [1]. A series of clinical case studies have reported a prevalence

*Correspondence: duanyy@sysucc.org.cn

†Zhihuan Zhou and Feifei Zuo contributed equally to this work.

1 State Key Laboratory of Oncology in South China, Collaborative Innovation Center for Cancer Medicine, and Guangdong Key Laboratory

of Nasopharyngeal Carcinoma Diagnosis and Therapy, Sun Yat-sen

University Cancer Center, Guangzhou, Guangdong, PR China

Full list of author information is available at the end of the article for PA among community-dwelling adults ranging from 1 in 865 to 1 in 2,688 [2]. The goals of PA surgery include the complete removal of the adenomas, the correction of hormonal hypersecretion, the retention of pituitary function, and the reduced risk of tumor recurrence [3, 4]. Compared with transcranial surgery, transsphenoidal surgery (TSS) is advantageous because it does not require brain retraction, resulting in fewer complications, shorter hospital stays, and better patient comfort [5]. With the evolution of imaging and surgical techniques, TSS has 
become an effective and preferred surgical approach for most PAs [4], associated with an extremely low mortality rate [3].

Although TSS for PA is considered safe [6], possible complications can occur. Postoperative cerebrospinal fluid (CSF) leakage represents a characteristic and potentially severe side effect [7] and remains a challenge even for experienced pituitary surgeons. Previous studies have reported that the incidence of CSF leakage after TSS ranged from $0.5 \%$ to $15 \%$ [7]. A national survey of complications after TSS found that although various repair methods have been developed, the incidence of postoperative CSF leakage remained high (3.9\%) [8]. If not treated properly, postoperative CSF leakage can lead to serious consequences, such as headache, incision infections, meningitis, and even encephalopyosis [9], which can be life-threatening, resulting in patients with enormous economic and psychological burdens [10].

Elucidating the risk factors associated with CSF leakage after TSS is important and helpful for patients with PA. The identification of these factors may permit the implementation of strategies to reduce postoperative complications. However, limited attention has been paid to factors associated with such CSF leaks. Some differences have been reported among the results of previous studies on risk factors, which may be due to different inclusion criteria and small sample sizes. No comprehensive meta-analysis has been performed to examine the risk factors for CSF leakage after TSS. Therefore, the purpose of our article was to systematically review the potential risk factors associated with the development of postoperative CSF leaks after TSS in patients with PA.

\section{Methods}

\section{Data sources}

A search was performed in PubMed, Web of Science, The Cochrane Library, Embase, China National Knowledge Infrastructure (CNKI), Wanfang database, and VIP database to identify articles published from database inception to December 2020. We used a search strategy that included truncated free text and exploded medical subject heading (MeSH) terms relevant to "pituitary adenoma," "postoperative cerebrospinal fluid leak," "transsphenoidal surgery," or "risk factors." For example, the search details for PubMed/Medline were as follows: ((("pituitary adenoma"[Title/Abstract] OR "pituitary adenoma"[All Fields] OR "pituitary tumor" [Title/Abstract] OR "pituitary tumor"[All Fields]) AND ("cerebrospinal fluid leak"[MeSH Terms] OR ("cerebrospinal"[All Fields] AND "fluid"[All Fields] AND "leak"[All Fields]) OR "cerebrospinal fluid leak"[All Fields])) AND ("transnasal”[Title/Abstract] OR "transnasal approach"[Title/Abstract] OR "transnasal approach"[All Fields] OR "transsphenoidal surgery"[Title/ Abstract] OR "transsphenoidal surgery" [All Fields]) AND "risk factor"). In addition, manual searches were conducted of the reference sections of retrieved articles to identify additional published work relevant to this study.

\section{Study selection}

All retrieved studies were reviewed for inclusion based on the following criteria: 1) patients who underwent TSS and were pathologically diagnosed with PA according to postoperative immunocytology; 2) studies mentioning CSF leakage after TSS; and 3) prospective or retrospective studies.

The exclusion criteria were as follows: 1) spontaneous CSF leakage; 2) case reports, reviews, letters, or dissertation; 3) articles in other languages than English and Chinese; 4) reported only duplicate data; 5) no data regarding postoperative CSF leakage; 6) cadaver or animal studies; and 7) articles with incomplete information or incomplete data.

Two reviewers independently screened the titles and abstracts of all articles for eligibility. The screening results from the two reviewers were compared, and disagreements were discussed to reach a consensus. Next, full-text reviews and data extraction were independently performed by the same two reviewers, and the results were again compared and discussed to reach an agreement. A third reviewer was consulted to assist in the resolution of unresolved discrepancies between reviewers at any stage in the article selection process.

\section{Data extraction and quality assessment}

Two authors independently extracted data from the selected articles using a standard data extraction form. Data included the following study items: publication year, first author, study design, sample size, population characteristics, number of CSF leaks after TSS, and associated risk factors.

Two authors independently assessed the quality of all included studies using the Newcastle-Ottawa Scale (NOS) [11]. The NOS assesses quality in terms of the selection of PA patients; the comparability of study groups, if applicable; and outcome assessments. Differences in quality assessments between the two authors were resolved discussion until consensus was reached. Studies with an NOS score $\geq 6$ that included appropriate statistical analyses were deemed to have high methodological quality.

\section{Statistical analyses}

Review Manager software (RevMan 5.3; Cochrane Collaboration, Oxford, United Kingdom) was used to 
perform statistical analyses. For each of the dependent factors, effect sizes were calculated separately. For each dependent variable, an overall effect size was calculated by weighting all the effect sizes calculated for each individual study according to relative sample sizes [12]. To test whether the variability in effect sizes exceeded what could be expected from sampling error alone, $\mathrm{I}^{2}$ tests for heterogeneity were conducted. When the $\mathrm{I}^{2}$ value was less than $50 \%$, indicating low heterogeneity, a fixed-effects analysis was used to estimate the assumed common effect. When the $\mathrm{I}^{2}$ value was $50 \%$ or above, indicating increased heterogeneity, a random-effects analysis was used to estimate the mean distribution of effects across all studies, yielding wider confidence intervals for the combined effect size [13]. In addition, a sensitivity analysis was performed by eliminating each included study one at a time when the heterogeneity was high $\left(\mathrm{I}^{2} \geq 50 \%\right)$. The odds ratios (ORs) and corresponding 95\% confidence intervals (95\% CIs) were calculated for categorical variables, based on the number of postoperative CSF leakage groups and the total sample size for each risk factor. Funnel plots were used to assess publication bias and were generated for all factors that were identified in more than 10 studies included in the present study [13].

\section{Results}

Literature search and study characteristics

A total of 14 case-control studies and 20 cohort studies were included in the present analysis. A flow diagram of the selection and exclusion processes, together with the respective justifications for exclusion, is shown in Fig. 1. After the application of inclusion and exclusion criteria, 34 articles were included in our quantitative analysis, and the characteristics of the 34 studies are summarized in Table 1. A total of 9,144 patients were enrolled in the meta-analysis, including 511 (5.6\%) who were diagnosed with postoperative CSF leakage.

\section{Risk factors for postoperative CSF leakage Tumor size}

A total of eight studies reported differences in the incidence of postoperative CSF leakage among patients with different tumor sizes (Fig. 2). The analysis of postoperative CSF leakage across tumor sizes revealed that giant adenoma was associated with a significantly increased risk of postoperative CSF leakage compared with macroadenoma or microadenoma (pooled OR: 3.18, 95\% CI: $1.20-8.38$ ). The sensitivity analysis suggested that when Huang's study [24] was excluded, the result (pooled OR: 4.57, 95\% CI: 2.31-9.05) was consistent with the overall result without excluding any

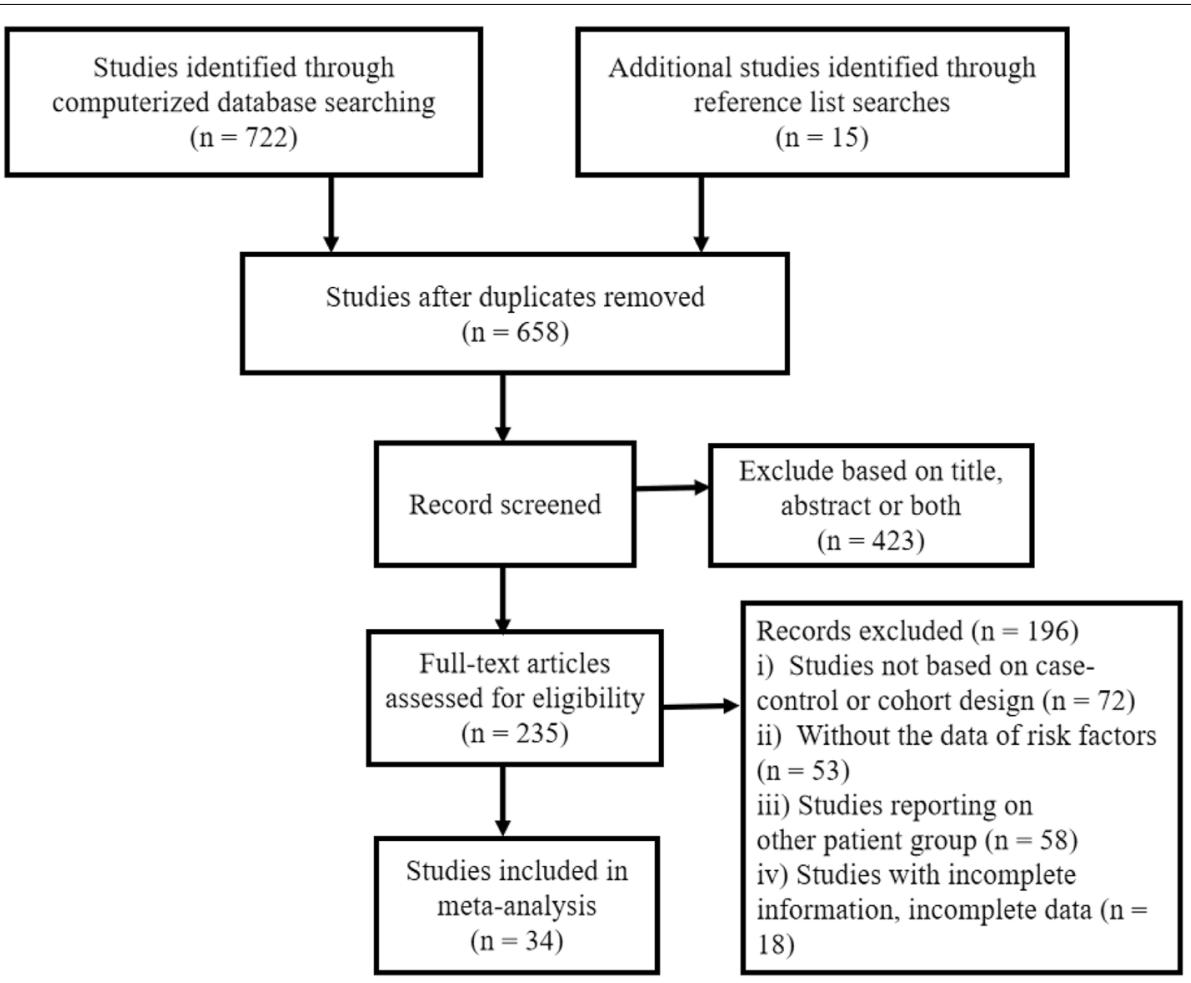

Fig. 1 Flow diagram of study search and criteria application 
Table 1 Baseline characteristics of included studies

\begin{tabular}{|c|c|c|c|c|c|c|}
\hline Study (first author) & Publication year & Study type & $\begin{array}{l}\text { No. of cases } \\
\text { with postop } \\
\text { CSF leakage }\end{array}$ & $\begin{array}{l}\text { Total no. of } \\
\text { Patients }\end{array}$ & Risk factors & NOS score \\
\hline $\mathrm{Xu}[14]$ & 2013 & case-control study & 64 & 1641 & 3,6 & 6 \\
\hline Cheng [15] & 2014 & case-control study & 9 & 129 & $1,2,3,4,6$ & 6 \\
\hline Tang [16] & 2018 & cohort study & 3 & 120 & 4 & 6 \\
\hline Zhao [17] & 2020 & cohort study & 40 & 158 & 5 & 8 \\
\hline Li [18] & 2016 & cohort study & 10 & 162 & 5 & 8 \\
\hline Liu [19] & 2020 & case-control study & 25 & 194 & $1,2,4,6$ & 8 \\
\hline Tian [20] & 2018 & case-control study & 29 & 1063 & $1,3,4,6$ & 8 \\
\hline Yin [21] & 2014 & cohort study & 6 & 81 & 5 & 8 \\
\hline Wang [22] & 2018 & case-control study & 11 & 112 & 4,6 & 8 \\
\hline Zhang [23] & 2017 & case-control study & 10 & 114 & $1,4,6$ & 8 \\
\hline Huang [24] & 2018 & case-control study & 33 & 270 & $1,2,4,6$ & 8 \\
\hline Ding [25] & 2019 & cohort study & 22 & 251 & 5 & 9 \\
\hline Liu [26] & 2014 & cohort study & 2 & 64 & 5 & 8 \\
\hline $\mathrm{He}[27]$ & 2015 & cohort study & 2 & 47 & 5 & 8 \\
\hline Wang [28] & 2011 & cohort study & 1 & 40 & 5 & 8 \\
\hline Yang [29] & 2017 & cohort study & 13 & 240 & 5 & 8 \\
\hline Wangx [30] & 2018 & cohort study & 11 & 64 & 5 & 8 \\
\hline Zhou [31] & 2015 & cohort study & 15 & 116 & 5 & 8 \\
\hline Liu [32] & 2017 & cohort study & 4 & 116 & 5 & 8 \\
\hline Chen [33] & 2012 & case-control study & 25 & 180 & $1,2,3$ & 6 \\
\hline Agam [34] & 2018 & cohort study & 30 & 1153 & 5 & 6 \\
\hline Fujimoto [35] & 2017 & case-control study & 8 & 162 & $1,4,6$ & 8 \\
\hline Gao [36] & 2016 & cohort study & 11 & 105 & 5 & 8 \\
\hline Guvenc [38] & 2016 & cohort study & 7 & 94 & 5 & 8 \\
\hline Han [9] & 2008 & case-control study & 26 & 592 & $1,2,3,4$ & 8 \\
\hline Karppinen [39] & 2015 & cohort study & 6 & 185 & 5 & 8 \\
\hline Liu [40] & 2019 & cohort study & 12 & 189 & 6 & 9 \\
\hline Mehta [41] & 2012 & cohort study & 8 & 158 & 6 & 8 \\
\hline Riesgo [37] & 2019 & case-control study & 7 & 302 & 3,4 & 6 \\
\hline Xue [42] & 2020 & case-control study & 13 & 216 & 4 & 6 \\
\hline Mansy [43] & 2010 & cohort study & 8 & 200 & 6 & 6 \\
\hline Zhangc [10] & 2017 & case-control study & 13 & 474 & 4,6 & 7 \\
\hline Dallapiazza [44] & 2014 & cohort study & 9 & 99 & 5 & 8 \\
\hline Thawani [45] & 2016 & case-control study & 21 & 203 & 4,6 & 9 \\
\hline
\end{tabular}

Risk Factors: 1. Tumor size 2. Consistency of adenoma 3. Revision surgery 4. Intraoperative CSF 5. Operation method 6. Other factors: Sex, functional adenoma type, resection rate or perioperative lumbar drainage

CSF cerebrospinal fluid, NOS Newcastle-Ottawa Scale

studies. However, the heterogeneity remained high $\left(\mathrm{I}^{2}\right.$ $=53 \%, P=0.05)$.

\section{Consistency of adenoma}

A total of five studies reported differences in the incidence of postoperative CSF leakage depending on the adenoma consistency (Fig. 3). The meta-analysis revealed that a hard tumor was associated with a significantly increased risk of postoperative CSF leakage compared with soft tumors (pooled OR: 3.20 , 95\% CI: 2.13-4.81).

\section{Primary vs. revision surgery}

A total of six studies provided data regarding the relationship between revision surgery and postoperative CSF leakage (Fig. 4). The meta-analysis revealed that revision 


\begin{tabular}{|c|c|c|c|c|c|c|c|c|c|}
\hline \multirow[b]{2}{*}{ Study or Subgroup } & \multicolumn{2}{|c|}{ Giant adenoma } & \multicolumn{3}{|c|}{ Macroadenoma/Microadenoma } & \multirow{2}{*}{$\begin{array}{l}\text { Odds Ratio } \\
\text { M-H. Random. } 95 \% \mathrm{Cl}\end{array}$} & \multirow{2}{*}{\multicolumn{3}{|c|}{$\begin{array}{l}\text { Odds Ratio } \\
\text { M-H. Random, } 95 \% \mathrm{Cl}\end{array}$}} \\
\hline & Events & Total & Events & Total & Weight & & & & \\
\hline Chen 2012 & 20 & 80 & 5 & 100 & $14.2 \%$ & $6.33[2.26,17.77]$ & &  & \\
\hline Cheng 2014 & 1 & 5 & 8 & 124 & $8.7 \%$ & $3.63[0.36,36.35]$ & & & \\
\hline Fujimoto 2017 & 2 & 3 & 1 & 9 & $6.0 \%$ & $16.00[0.67,383.02]$ & & & \\
\hline Han 2008 & 14 & 154 & 12 & 438 & $15.2 \%$ & $3.55[1.60,7.86]$ & & & \\
\hline Huang 2018 & 5 & 89 & 28 & 181 & $14.4 \%$ & $0.33[0.12,0.87]$ & & & \\
\hline Liu 2020 & 11 & 18 & 14 & 176 & $13.9 \%$ & $18.18[6.09,54.28]$ & & & \\
\hline Tian 2018 & 6 & 83 & 23 & 974 & $14.7 \%$ & $3.22[1.27,8.15]$ & & $\Rightarrow$ & \\
\hline Zhang 2017 & 4 & 44 & 6 & 70 & $12.9 \%$ & $1.07[0.28,4.01]$ & & & \\
\hline Total $(95 \% \mathrm{Cl})$ & & 476 & & 2072 & $100.0 \%$ & $3.18[1.20,8.38]$ & & & \\
\hline Total events & 63 & & 97 & & & & & & \\
\hline \multirow{2}{*}{\multicolumn{7}{|c|}{$\begin{array}{l}\text { Heterogeneity: } \text { Tau }^{2}=1.45 ; \mathrm{Ch}^{2}=36.60, \mathrm{df}=7(\mathrm{P}<0.00001) ; \mathrm{I}^{2}=81 \% \\
\text { Test for overall effect: } Z=2.34(P=0.02)\end{array}$}} & & & 100 \\
\hline & & & & & & & $\begin{array}{l}0.01 \quad 0.1 \\
\text { Favours [experimental] }\end{array}$ & Favours [control] & \\
\hline
\end{tabular}

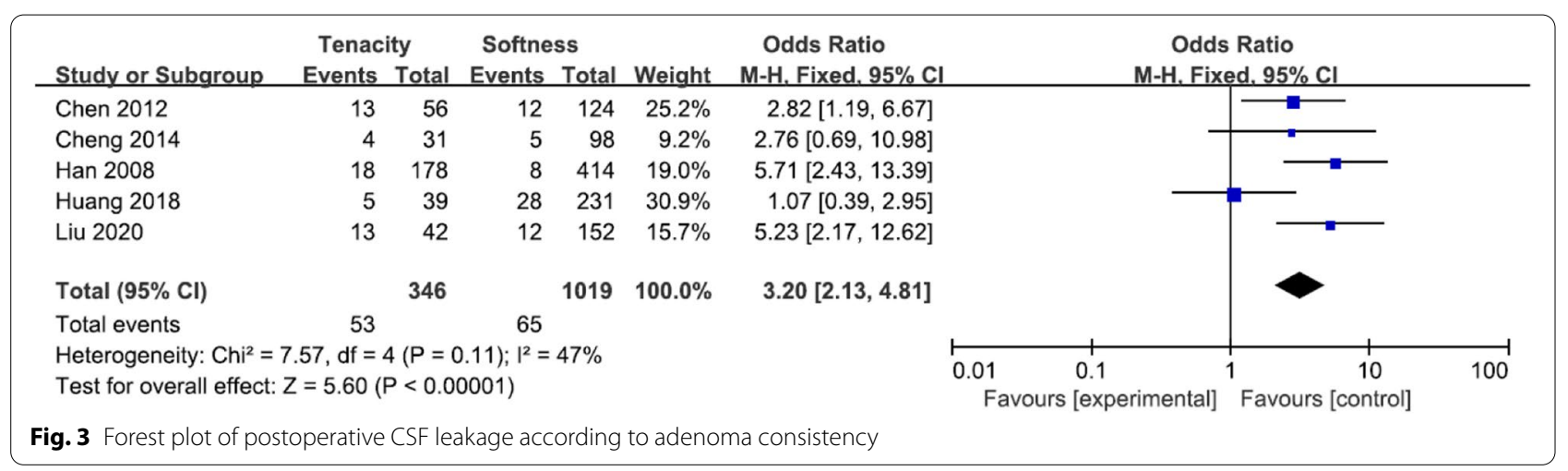

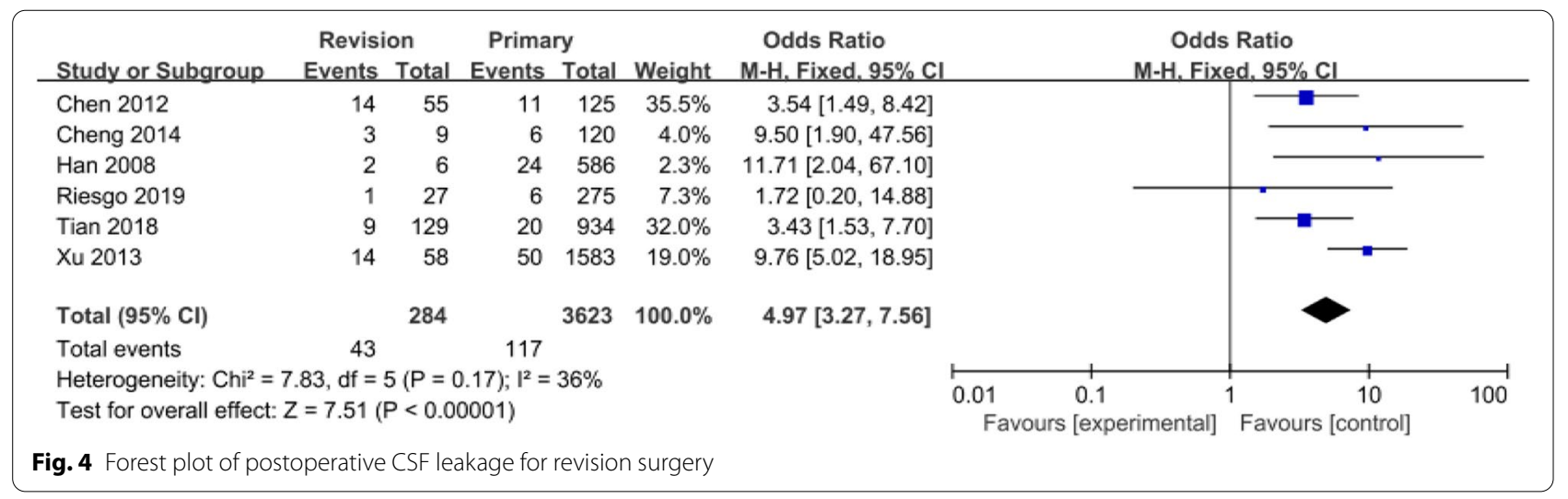

surgery was associated with a significantly increased risk of postoperative CSF leakage compared with primary surgery (pooled OR: 4.97, 95\% CI: 3.27-7.56).

\section{CSF during the operation (intraoperative CSF)}

A total of 13 studies provided data regarding the relationship between intraoperative CSF leakage and postoperative CSF leakage (Fig. 5a). The meta-analysis revealed that intraoperative CSF leakage was associated with a significantly increased risk of postoperative CSF leakage (pooled OR: 6.33, 95\% CI: 3.67-10.92) relative to no CSF leakage. The sensitivity analysis suggested that when Tian's study [20] was excluded, the results remained stable (pooled OR: 5.33, 95\% CI: 3.60-7.90), and the heterogeneity was reduced $\left(\mathrm{I}^{2}=21 \%, P>0.05\right)$. Through discussion among the researchers, Tian's study was determined to meet the inclusion criteria and was retained. Using a funnel plot, most studies were found to be distributed in the center and top of the plot, indicating little publication bias (Fig. 5b). 


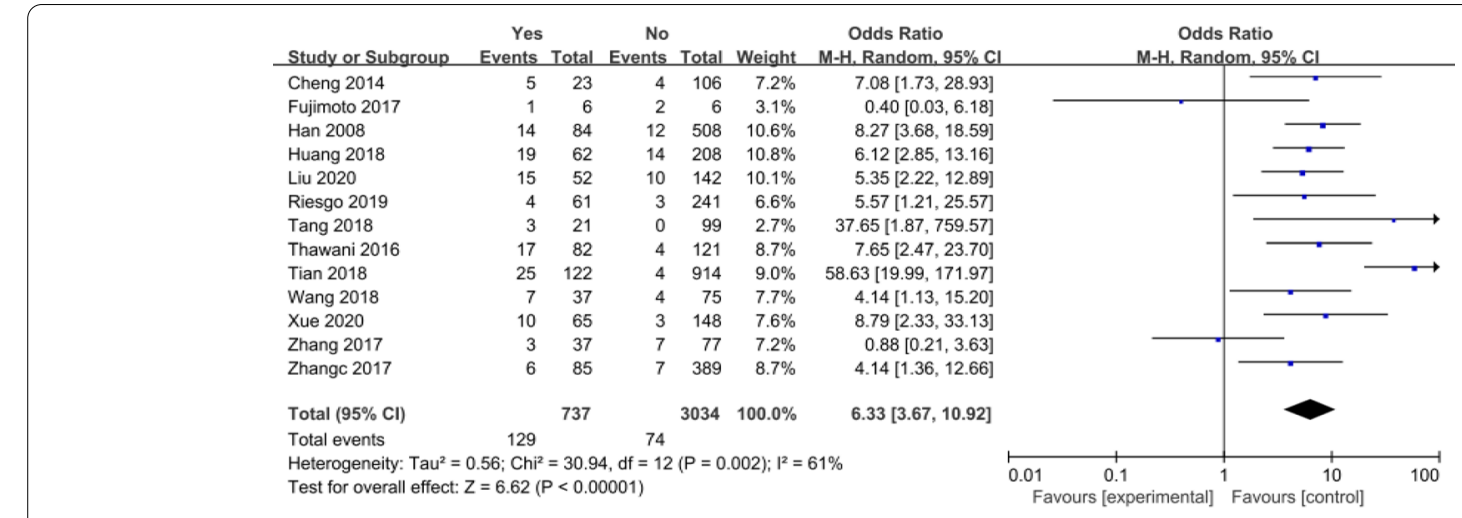

Odds Ratio

\section{a. Forest plot of postoperative CSF leakage according to the occurrence}

of intraoperative CSF leakage

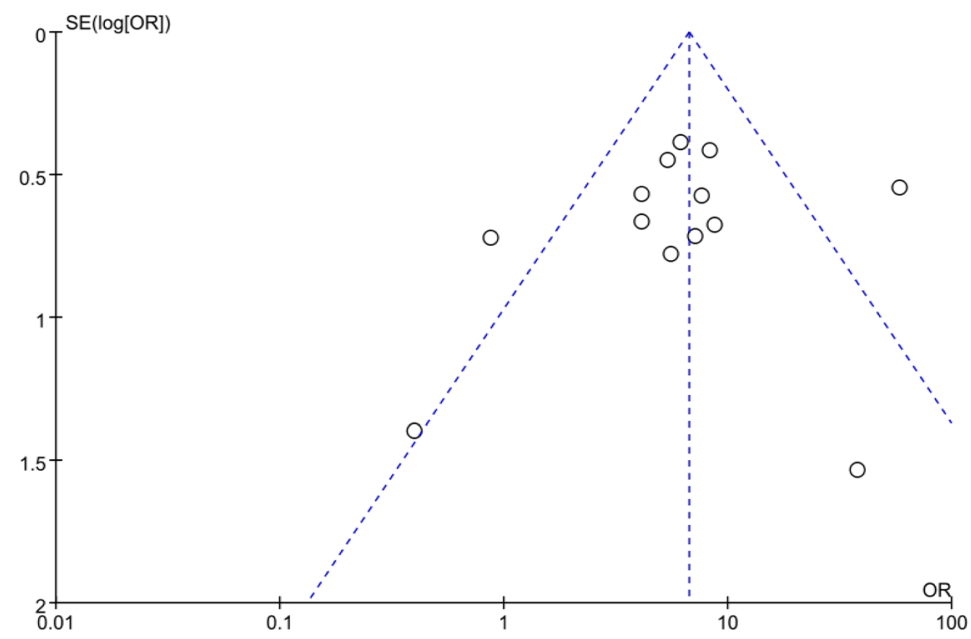

b. Funnel plot of postoperative CSF leakage for the occurrence of

intraoperative CSF leakage

Fig. 5 a Forest plot of postoperative CSF leakage according to the occurrence of intraoperative CSF leakage. b Funnel plot of postoperative CSF leakage for the occurrence of intraoperative CSF leakage

\section{Operation method}

A total of 16 studies reported differences in the incidence of postoperative CSF leakage between the endoscopic approach and the microscopic approach for TSS (Fig. 6a). The meta-analysis revealed that the endoscopic approach was associated with a significantly reduced risk of postoperative CSF leakage compared with the microscopic approach (pooled OR: 0.69, 95\% CI: 0.50-0.96). In the generated funnel plot, most studies were distributed in the center and top of the plot, indicating little publication bias (Fig. 6b).

\section{Other factors}

The meta-analysis suggested no significant difference in the incidence of postoperative CSF leakage across sexes (pooled OR: 1.78, 95\% CI: 1.00-3.14) or functional adenoma types (pooled OR: 1.32, 95\% CI: 0.84-2.05). Similarly, total resection (pooled OR: 0.77 , 95\% CI: $0.21-$ 2.80 ) and no perioperative lumbar drainage (pooled OR: 0.61, 95\% CI: $0.18-2.11$ ) are not risk factors for postoperative CSF leakage. We have included these non-significant factors analyzed and the related diagrams in a "Supplementary Materials".

\section{Discussion}

This review quantitatively synthesized the currently available evidence on the prevalence and risk factors associated with the incidence of postoperative CSF leakage after TSS in 34 studies, which included 9,144 participants with $\mathrm{PA}$, to demonstrate a pooled global prevalence of $5.6 \%$. 


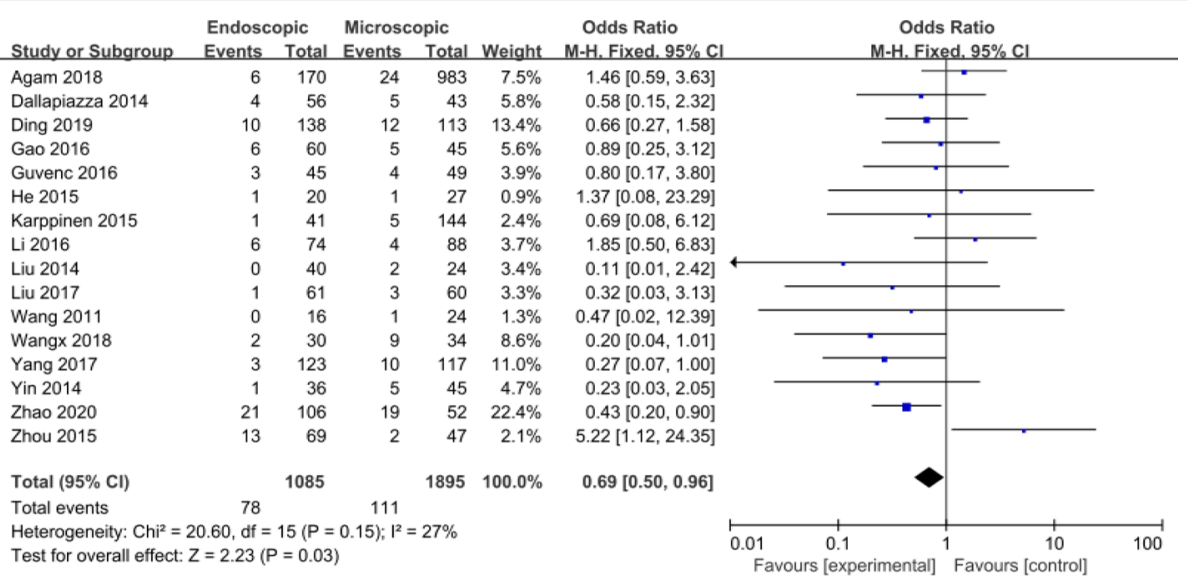

\section{a. Forest plot of postoperative CSF leakage according to the operation} method

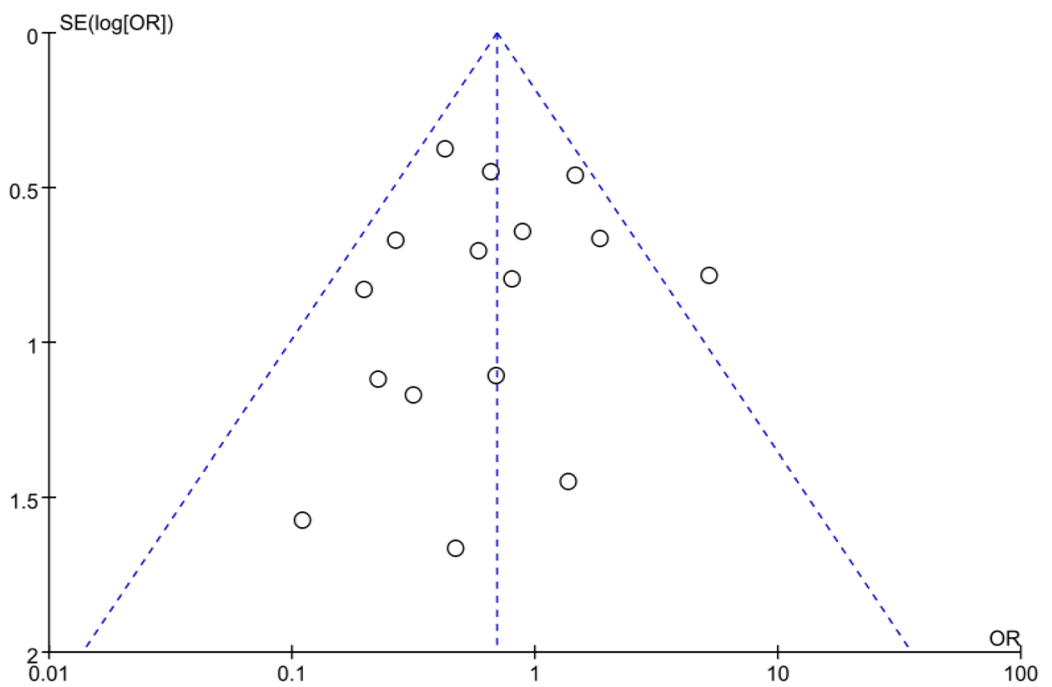

\section{b. Funnel plot of postoperative CSF leakage based on Operation method}

Fig. 6 a Forest plot of postoperative CSF leakage according to the operation method. b Funnel plot of postoperative CSF leakage based on Operation method

We evaluated the relationships between these nine factors and the risk of postoperative CSF leakage. Our data indicated that tumor size, adenoma consistency, revision surgery, and intraoperative CSF leakage were independent risk factors for postoperative CSF leakage. By contrast, the use of an endoscopic approach showed a significant protective benefit compared with the microscopic approach in TSS, whereas sex, functional adenoma type, resection rate, and perioperative LD were not found to be related to the occurrence of postoperative CSF leakage.

The effect of tumor size on postoperative CSF leakage is under debate, with some studies showing that patients with giant adenoma are more likely to experience postoperative CSF leakage than those with macroadenoma or microadenoma $[9,10]$. In contrast, Shiley et al. [46] and Nishioka et al. [47] found that CSF leakage was more common among patients with microadenomas. In addition, Chi et al. [48] reported that tumor size was not associated with postoperative CSF leakage. Han et al. [9] indicated that large pituitary tumors commonly expand the sella and erode regions adjacent to the meninges, resulting in the attenuation of these barriers to the CSF space. Moreover, larger pituitary tumors result in the wider invasion of adjacent tissues, resulting 
in a greater extent of resection, which may increase the risk of CSF leakage after TSS [19]. In our study, tumor size was identified as a risk factor for postoperative CSF leakage, with high heterogeneity. Therefore, more highquality research is necessary to better explore the effects of tumor size on postoperative CSF leakage occurrence.

The current analysis showed that adenoma consistency was associated with the occurrence of postoperative CSF leakage. Most PAs have a soft texture that can easily be resected with curettage and suction [49]. Hard (fibrous) tumors account for approximately $5 \%-13 \%$ of PA and are difficult to separate from critical structures, often requiring removal using sharp dissection or laser techniques [9, 49]. Therefore, the consistency of an adenoma affects its resection success [50]. In our meta-analysis, compared with soft tumors, firm pituitary tumors were found to be associated with a greater risk of CSF leakage after TSS, with crude ORs of 2.13-4.81. Therefore, the PA consistency must be assessed using reliable imaging techniques (such as magnetic resonance imaging, MRI) before performing TSS, which may help surgeons to better plan an appropriate operative strategy and reduce the risk of surgery.

A strong association was observed between revision surgery and an increased risk of postoperative CSF leakage, which is consistent with the findings of a previous study [9, 46, 47]. Shiley et al. [46] found that the incidence of postoperative CSF leakage after revision surgery was significantly higher than that after primary surgery (14.6\% vs. $4.0 \%, \mathrm{P}=0.010)$ in a study examining 235 patients undergoing TSS. The first procedure can create scar tissue that adheres to the arachnoid space and diaphragm [51]. The revision surgery is made more difficult by the presence of adhesions, tissue fibrosis, abnormal vascular hyperplasia, and distorted anatomy, which can increase the complexity of the dissection [20]. Moreover, revision surgery requires the removal of residual tumors that were not easily detected or resectable during the prior surgery, which typically requires a more aggressive dissection approach along the sellar diaphragm, increasing the risk of postoperative CSF leakage [20].

In our results, patients with an intraoperative CSF leak were 6.33 times more likely to experience postoperative CSF leakage than patients without intraoperative CSF leakage. Seiler et al. [52] found that the occurrence of postoperative CSF leakage was six times as common among cases that reported intraoperative CSF leakage compared with those that did not. Similar results were observed in a retrospective analysis, which showed a significant difference in the rates of postoperative CSF leakage between patients who experienced an intraoperative CSF leak and those who did not (16.7\% vs. $2.3 \%)$ [9]. Not surprisingly, intraoperative CSF leakage was correlated with the risk of postoperative CSF leakage, which may be due to the incomplete repair of intraoperative CSF leakage [20]. The unidentified or delayed development of intraoperative CSF leaks is also an equally important source of postoperative CSF leakage as the failure to employ effective CSF leak repair methods [53]. In addition, repair materials may shift or fall off due to postoperative actions that increase intracranial pressure (such as sneezing and constipation), resulting in incomplete leakage closure. Sarita et al. [54] found that chronic cough was one of the primary contributing factors to the failure to resolve an intraoperative leak, leading to the postoperative recurrence of the leak. Our findings suggested that patients with intraoperative CSF leaks may warrant more aggressive management to prevent the development of postoperative leaks, and correct behavior education is also important for the management of postoperative CSF leakage.

PAs with high levels of invasiveness are more likely to invade the peritumoral tissues, such as the cavernous sinus and the internal carotid artery, which may increase the difficulty of TSS. To completely remove the tumor, the surgeon may overstretch the sellar septum and dura mater, which increases the risk of CSF leakage [14]. In addition, the arachnoid membrane at the sellar diaphragmatic foramen can be easily damaged when most of the tissue around the PA is removed, resulting in CSF leakage. In our meta-analysis, few studies focused on the effects of invasiveness of PA on the occurrence of postoperative CSF leakage; therefore, we did not confirm whether this variable may serve as a clinically useful marker of an individual's propensity toward postoperative CSF leakage. However, in cases of PA with high invasiveness, more attention should be given to local anatomy and surgical skill [55]. To prevent the occurrence of CSF leakage during TSS, the PA should be removed according to appropriate procedures, and the traction of the peritumoral tissues should be reduced as much as possible to avoid causing damage to the sellar septum and dura mater.

The current study found a mild association between the performance of endoscopic TSS and a reduced risk of postoperative CSF leakage, with crude ORs of 0.50-0.96. A meta-analysis of 23 observational studies conducted by Li et al. [56] previously showed that endoscopic TSS had no significant effect on the risk of CSF leakage compared with microscopic TSS. However, the data analyzed by Li et al. [56] cannot be directly compared with our series because their results were not limited to postoperative CSF leakage and encompassed all pituitary pathology. In addition, relatively few complications were reported. Compared with the microscopic approach, the endoscopic approach has many advantages. It is easier, 
requires a less traumatic entry into the sphenoid sinus, and enables wide and close views of the tumor, allowing for increased tumor resection [38]. By contrast, some surgeons believe that the endoscopic approach decreases stereoscopic visualization and decreases the ability to use their instruments [57], which may account for the lack of significant difference observed for the incidence of CSF leakage between these two surgical techniques in some studies [34, 38]. In our study, the endoscopic approach showed a minimally protective benefit for reducing postoperative CSF leakage compared with the microscopic approach. Surgeons must be specially trained for an endoscopic approach [38], and the surgeon's learning curve was found to be associated with the occurrence of postoperative CSF leaks [58]. The results of endoscopic TSS may improve with the experience of individual surgeons. Therefore, further research remains necessary to explore the potentially protective role of using the endoscopic approach to prevent the occurrence of postoperative CSF leakage.

Although sex, aging, and body mass index (BMI) were reportedly associated with postoperative CSF leakage [58], the current evidence could not be used to determine whether these demographic factors were risk factors. A multi-institutional study of patients undergoing endoscopic PA showed that the risk of postoperative CSF leakage in female patients was 2.4 times higher than that in male patients [59]. However, Zhang et al. [23] and Tian et al. [20] have shown that the female sex of patients had no effect on the occurrence of postoperative CSF leakage. Whether sex is a risk factor for postoperative CSF leakage requires further investigation. Relatively few studies have examined the impacts of aging and BMI on postoperative CSF leakage in PA patients undergoing TSS, and various definitions of aging and BMI were used in these studies, making the meta-analysis of these two factors difficult to perform. Caitlin et al. [59] found that younger patients $(<64$ years) had a higher risk of postoperative CSF leakage than those older than 64 years. In two retrospective analyses, no correlation was observed between average patient age and the occurrence of postoperative CSF leakage $[46,47]$. Several studies showed that an elevated BMI was an independent predictor of postoperative CSF leakage after TSS $[59,60]$. This association might be due to the increased intra-abdominal pressure associated with higher BMI [58]. A moderate level of physical activity (e.g., 150 minutes of moderate aerobic exercise) for BMI reduction may be helpful [61]. In addition, more studies remain necessary to quantify the effects of aging and BMI on the occurrence of postoperative CSF leakage in PA patients undergoing TSS.

The present meta-analysis could not determine whether the functional adenoma type was associated with an increased risk of postoperative CSF leakage in PA patients undergoing TSS. Two studies found that postoperative CSF leakage was more common in adrenocorticotropic hormone $(\mathrm{ACTH})$-producing adenomas [46, 47], which is likely due to ACTH adenomas often not appearing localized on preoperative imaging and requiring a more aggressive resection approach $[46,47]$. However, only a few events of postoperative CSF leakage were reported in these studies, and the conclusions were prone to bias. Han et al. [9] found that the postoperative leakage rates associated with follicle-stimulating hormone (FSH) adenomas were higher than those for other tumor types. By contrast, Tian et al. [20] and Wang et al. [22] found that the functional adenoma types were not a significant risk factor for postoperative CSF leakage; therefore, this inconsistency requires further study.

Whether the resection rate is a risk factor for postoperative CSF leakage remains controversial. A retrospective analysis of 1,641 patients with PA undergoing TSS showed that the incidence of postoperative CSF leakage in patients with total resection was significantly higher than that among patients with partial or subtotal resections [14]. The mechanism underlying this association may be that tumors that undergo total resection, especially for giant or invasive adenomas, experience more severe dural stretch, increasing the risk of postoperative CSF leakage [14]. The literature exploring the resection rate remains scarce and reported results have been conflicting and highly heterogeneous. Further studies must be performed to confirm the relationship between the resection rate and the occurrence of postoperative CSF leakage.

The use of LD remains under debate. In a meta-analysis [5], LD had an OR 1.13 for reducing the occurrence of postoperative CSF leakage after endonasal endoscopic skull base surgery. Gautam et al. [41] posited that preoperative or intraoperative LD could reduce tension on the arachnoid caused by the expansion of a pituitary macroadenoma, preventing the potential exposure of the arachnoid to intraoperative injury and reducing the risk of intraoperative CSF leakage. However, Jung et al. [5] suggested that these results should be interpreted carefully because surgeons tend to perform LD in high-risk patients or when the surgeon feels that the reconstruction was not completely successful. A prospective study of 114 TSS procedures for pituitary macroadenoma indicated that LD reduced the rate of intraoperative CSF leakage from 41 to $5 \%(\mathrm{p}<0.001)$, but the rate of postoperative CSF leakage remained similar (5 vs. $5 \%$ ). In addition, some researchers have stated that the presence of the drain may mask the earlier detection of a CSF leak [43]. Therefore, whether LD reduces the risk of postoperative CSF leakage after TSS remains inconclusive given the current evidence. 
The acknowledged limitations of this study should be mentioned. First, unpublished articles were not included in this systematic review, likely contributing to publication bias. Second, a lack of randomized control trials (RCTs) examining the occurrence of postoperative CSF leakage exists, which may reduce the reliability of the results of our study. Further studies using RCT designs may enrich and substantiate our results. Moreover, there were many kinds of fillers, such as gelfoam, absorbable hemostatic gauze, autologous fat, pedicled vascularized flap and so on. A specialized mesh meta-analysis is better used to compare which closure techniques can reduce the risk of CSF leakage. Despite these limitations, this study represents the first known meta-analysis to examine the risk factors associated with postoperative CSF leakage after TSS in PA patients.

This study makes a significant contribution to clinical practice because our findings can focus clinical medical workers' attention on the occurrence of postoperative CSF leakage after TSS in PA patients. We have shown that which factors are more likely to cause CSF leakage in these patients for clinicians, especially neurosurgeons. When operating on PA patients with high-risk CSF leakage, surgeons should take some targeted strategies, such as selecting appropriate surgical methods and resection techniques. In addition, clinical nurses should strengthen observation of high-risk patients in postoperative nursing, (such as more frequent ward rounds), to achieve early detection and treatment of CSF leakage.

\section{Conclusion}

This review provides a comprehensive overview of the quality of the evidence base to inform clinical staff of the importance of screening risk factors for postoperative CSF leakage after TSS for PA. The risk factors associated with postoperative CSF leakage after TSS include tumor size, adenoma consistency, revision surgery, and intraoperative CSF leakage. However, the use of the endoscopic approach showed a significant protective benefit compared with the microscopic approach in TSS, whereas sex, adenoma functional type, the resection rate, and perioperative LD were not identified as significant risk factors for CSF leakage in this meta-analysis. More attention should be paid to PA patients with high risks of CSF leakage after TSS to reduce complications and improve prognosis.

\section{Supplementary Information}

The online version contains supplementary material available at https://doi. org/10.1186/s12883-021-02440-0.

Additional file 1.
Acknowledgements

The authors thank Meng Yang, for his assistance with statistics.

\section{Authors' contributions}

All authors contributed to the study conception and design. Material preparation, data collection and analysis were performed by Z. Zhou, F. Zuo, and Y. Duan. The first draft of the manuscript was written by Z. Zhou, F. Zuo, and Y. Duan, and all authors commented on previous versions of the manuscript. All authors read and approved the final manuscript.

\section{Funding}

This research was partly supported by grants from the National Natural Science Foundation of China for Young Scholars (No: 81702479).

Availability of data and materials

Not applicable.

\section{Declarations}

Ethics approval and consent to participate

Ethics committee or institutional review board approval was not required for systematic reviews and meta-analysis.

Consent for publication

Not applicable.

Competing interests

The authors have no relevant financial or non-financial interests to disclose.

\section{Author details}

${ }^{1}$ State Key Laboratory of Oncology in South China, Collaborative Innovation Center for Cancer Medicine, and Guangdong Key Laboratory of Nasopharyngeal Carcinoma Diagnosis and Therapy, Sun Yat-sen University Cancer Center, Guangzhou, Guangdong, PR China. ²Department of Neurosurgery, Sun Yat-sen University Cancer Center, Guangzhou, Guangdong, PR China. ${ }^{3}$ Department of Nasopharyngeal Carcinoma, Sun Yat-sen University Cancer Center, 651

Dongfeng Road East, Guangzhou 510060, PR China.

Received: 8 June 2021 Accepted: 12 October 2021

Published online: 27 October 2021

\section{References}

1. Lake MG, Krook LS, Cruz SV. Pituitary adenomas: an overview. Am Fam Physician. 2013;88(5):319-27.

2. Molitch ME. Diagnosis and treatment of pituitary adenomas: a review. JAMA. 2017:317(5):516-24. https://doi.org/10.1001/jama.2016.19699.

3. Couldwell WT. Transsphenoidal and transcranial surgery for pituitary adenomas. J Neuro-Oncol. 2004;69(1-3):237-56. https://doi.org/10. 1023/b:neon.0000041886.61149.ab.

4. Joshi SM, Cudlip S. Transsphenoidal surgery. Pituitary. 2008;11(4):353-60. https://doi.org/10.1007/s11102-008-0094-6.

5. Kim JS, Hong SD. Risk factors for postoperative CSF leakage after endonasal endoscopic skull base surgery: a meta-analysis and systematic review. Rhinology. 2021;59(1):10-20. https://doi.org/10.4193/Rhin20.145.

6. Singh H, Essayed WI, Cohen-Gadol A, Zada G, Schwartz TH. Resection of pituitary tumors: endoscopic versus microscopic. J Neuro-Oncol. 2016;130(2):309-17. https://doi.org/10.1007/s11060-016-2124-y.

7. Dallapiazza RF, Jane JJ. Outcomes of endoscopic transsphenoidal pituitary surgery. Endocrinol Metab Clin N Am. 2015;44(1):105-15. https:// doi.org/10.1016/j.ecl.2014.10.010.

8. Ciric I, Ragin A, Baumgartner C, Pierce D. Complications of transsphenoidal surgery: results of a national survey, review of the literature, and personal experience. Neurosurgery. 1997;40(2):225-36, 236-237. https://doi.org/10.1097/00006123-199702000-00001.

9. Han ZL, He DS, Mao ZG, Wang HJ. Cerebrospinal fluid rhinorrhea following trans-sphenoidal pituitary macroadenoma surgery: experience from 592 patients. Clin Neurol Neurosurg. 2008;110(6):570-9. https:// doi.org/10.1016/j.clineuro.2008.02.017. 
10. Zhang C, Ding X, Lu Y, Hu L, Hu G. Cerebrospinal fluid rhinorrhoea following transsphenoidal surgery for pituitary adenoma: experience in a Chinese centre. Acta Otorhinolaryngol Ital. 2017;37(4):303-7. https:// doi.org/10.14639/0392-100X-1086.

11. Wells G. (The Newcastle-Ottawa Scale (NOS) for Assessing the Quality of Non-Randomised Studies in Meta-Analyses. Symposium on Systematic Reviews: Beyond the Basics, 2014

12. de Ruiter MA, van Mourik R, Schouten-Van MA, Grootenhuis MA, Oosterlaan J. Neurocognitive consequences of a paediatric brain tumour and its treatment: a meta-analysis. Dev Med Child Neurol. 2013;55(5):408-17. https://doi.org/10.1111/dmcn.12020.

13. Higgins JP, Green S (2009) Cochrane Handbook for Systematic Reviews of Interventions. Naunyn Schmiedebergs Archiv Für Experimentelle Pathologie Und Pharmakologie.

14. Xu W, Rong W, Yong H, He X, Hu G. Relative factor analysis and prevention of cerebrospinal fluid rhinorrhea after monorhinic transsphenoidal pituitary tumor resection[In Chinese]. Chongqing Med. 2013;20(42):2366-8. https://doi.org/10.3969/j.issn.1671-8348.2013.20. 022.

15. Cheng Y, Ze-Qing LI, Zhang Y, Liu KD, Zhou M, Wang ZY, et al. Risk factors in cerebrospinal fluid rhinorrhea following transnasosphenoidal resection of pituitary adenoma[In Chinese]. Chin J Otorhinolaryngol Skull Base Surg. 2014;20(02):134-6.

16. Tingjun T. The causes analysis and treatment of main complications after transsphenoidal surgery for pituitary adenoma[ln Chinese]: Zunyi Medical University; 2018.

17. Yajun Z, Pingyang L, Zhonghua W, Tianxiao L, Bin W, Yongming L. Comparison of transnasał-sphenoidal ectomy of pituitary adenoma by microscope and neuroendoscope[In Chinese]. Chinese J Pract Nerv Dis. 2020;23(3):236-40.

18. Ming-Jian LI, Wang DK, Wang YT, Sun NB, Qiu CL, Surgery DO, et al. Clinical Effect Analysis of Transnasal-transsphenoidal Endoscopic Surgery and Microscopic Surgery of Pituitary Adenoma[In Chinese]. Acta Acad Med Weifang. 2016;5(38):391-3. https://doi.org/10.16846/j.issn. 1004-3101.2016.05.023.

19. Qi L, Yaosai L, Jun L, Zhong W, Yang C, Yihao W, et al. Analysis of risk factors related to cerebrospinal fluid leak due to endoscopic transnasal surgery for pituitary adenoma[In Chinese]. J Clin Neurosurg. 2020;17(5):516-21. https://doi.org/10.3969/j.issn.1672-7770.2020.05 008.

20. Weidong T, Xianghui M, Tao Z, Peng W, Fuyu W, Xinguang $Y$, et al. Risk factors for cerebrospinal fluid fistulas due to endoscopic transnasal surgery for pituitary neoplasms[In Chinese]. Chin J Neuromed. 2018;17(6):563-9. https://doi.org/10.3760/cma.j.issn.1671-8925.2018.06.005.

21. Huiyong Y. Clinical contrast study of endonasal endoscopic surgery andmicrosurgery for pituitary adenoma[In Chinese]: Zhengzhou University; 2014.

22. Ming W, Yong P, Yugang J. Risk factors of cerebrospinal fluid leak in endoscopic endonasal transsphenoidal pituitary surgery[ln Chinese]. Chin J Neurosurg. 2018;34(3):277-81. https://doi.org/10.3760/cma.jissn. 1001-2346.2018.03.013.

23. Zhongyuan Z. Analysis complications and relative risk factors after endoscopic resection of pituitary adenoma[In Chinese]: Zhejiang University; 2017.

24. Zhenxing $\mathrm{H}$. An analysis of risk factors for postoperative complications in endoscopic endonasal pituitary surgery[In Chinese]: Shandong University; 2018.

25. Zhiquan D. Clinical research of neuroendoscopic and microscopic transsphenoidal approach for the resection of nonfunctional pituitary adenomas[In Chinese]: Southern Medical University; 2019.

26. Liu Y, Yong Z, Cheng Z, Wang J, Wu Y, Cheng Z, et al. Comparison of curative effect of resection of pituitary adenoma by endoscope and microscope single nostril transsphenoidal approach[In Chinese]. Chinese J Brain Dis Rehabil. 2014;6:31-5. https://doi.org/10.3877/cma.j.issn.2095123X.2014.06.006

27. He H, Feng C, Wang W, Neurosurgery DO. Comparison of neuroendoscopy and microscopy for transnasal resection of giant pituitary adenomas[In Chinese]. Anhui Med J. 2015;7:805-8. https://doi.org/10. 3969/j.issn.1000-0399.2015.07.008

28. Wang F, Li A, Sun W. Comparison of neuroendoscopy and microscopy for transnasal resection of pituitary tumors[In Chinese]. Chinese J Minim
Invasive Surg. 2011;6(16):547-50. https://doi.org/10.3969/j.issn.10096604.2011 .06 .020

29. Yang Z, Wei X, Xu R, Neurosurgery DO. Contrast study of clinical efficacy and safety between endoscopic and microscopic surgery via transnasaltranssphenoidal approach for pituitary adenoma[In Chinese]. Henan Med Res. 2017;3(26):402-5. https://doi.org/10.3969/j.issn.1004-437X.2017.03.006.

30. Wang XD, Tang YY, Liu HQ, Neurosurgery DO. Comparison of neuroendoscopic and transnasal-sphenoidal microscopic resection of pituitary adenoma[In Chinese]. J Clin Neurosurg. 2018;4(15):292-5. https://doi.org/ 10.3969/j.issn.1672-7770.2018.04.011.

31. Zhou H, Wang ZM, Jiu-Jun PU, Zhong AL, Ruan LL, Jin K, et al. Comparative analysis of neuroendoscopic and microscopic trans-nasal-sphenoidal giant pituitary adenomas resection[In Chinese]. China J Endosc. 2015:8(21):785-9.

32. Yan L. Clinical contrast study of endonasal endoscopic surgery and microsurgery for pituitary adenoma[In Chinese]: Jilin University; 2017.

33. Dong C, Hong Y. The analysis of prognostic factors of cerebrospinal fluid leakage in the endoscopic sinus surgery[In Chinese]. China Medi Herald. 2012;9(28):159-60. https://doi.org/10.3969/j.issn.1673-7210.2012.28.068.

34. Agam MS, Wedemeyer MA, Bozena W, Weiss MH, Carmichael JD, Gabriel Z. Complications associated with microscopic and endoscopic transsphenoidal pituitary surgery: experience of 1153 consecutive cases treated at a single tertiary care pituitary center. J Neurosurg. 2018;5(130):1576-83. https://doi.org/10.3171/2017.12.JNS172318.

35. Fujimoto K, Yano S, Shinojima N, Hide T, Kuratsu JI. Endoscopic endonasal transsphenoidal surgery for patients aged over 80 years with pituitary adenomas: Surgical and follow-up results. Surg Neurol Int. 2017;8(1):213. https://doi.org/10.4103/sni.sni_189_17.

36. Yang G, Hui Z, Xu S, Yan Z, Zhong C. Endoscopic versus microscopic approach in pituitary surgery. J Craniofac Surg. 2016;2(27):157-9. https:// doi.org/10.1097/SCS.0000000000002401.

37. Riesgo P, Marino P, Platero A, Tarazona FJ, Fajardo C, Llacer JL, et al. Postoperative CSF leakages after transsphenoidal surgery for pituitary adenomas: analysis of a series of 302 surgical procedures. Neurocirugia. 2019:30(5):215-21. https://doi.org/10.1016/..neucir.2019.03.003.

38. Guvenc G, Kizmazoglu C, Pinar E, Imre A, Kaya I, Bezircioglu H, et al. Outcomes and complications of endoscopic versus microscopic transsphenoidal surgery in pituitary adenoma. J Craniofac Surg. 2016;27(4):1015-20.

39. Karppinen A, Kivipelto L, Vehkavaara S, Ritvonen E, Tikkanen E, Kivisaari R, et al. Transition from microscopic to endoscopic transsphenoidal surgery for nonfunctional pituitary adenomas. World Neurosurg. 2015;84(1):4857. https://doi.org/10.1016/j.wneu.2015.02.024.

40. Liu B, Wang Y, Zheng T, Liu S, Lv W, Lu D, et al. Effect of intraoperative lumbar drainage on gross total resection and cerebrospinal fluid leak rates in endoscopic transsphenoidal surgery of pituitary macroadenomas. World Neurosurg. 2019;135:629-39. https://doi.org/10.1016/j.wneu.2019.12.100.

41. Mehta GU, Oldfield EH. Prevention of intraoperative cerebrospinal fluid leaks by lumbar cerebrospinal fluid drainage during surgery for pituitary macroadenomas. J Neurosurg. 2012;116(6):1299-303. https://doi.org/10. 3171/2012.3.JNS112160.

42. Xue H, Wang X, Yang Z, Bi Z, Liu P. Risk factors and outcomes of cerebrospinal fluid leak related to endoscopic pituitary adenoma surgery. $\mathrm{Br} J$ Neurosurg. 2020;34(4):447-52. https://doi.org/10.1080/02688697.2020. 1754336.

43. Mansy A, Kersh A, Eissa E. Role of the external lumbar drain in management of CSF leak during or after transsphenoidal surgery. Egypt I Neurol Psychiatry Neurosurg. 2010;47(3):483-8.

44. Dallapiazza R, Bond AE, Grober Y, Louis RG, Payne SC, Oldfield EH, et al. Retrospective analysis of a concurrent series of microscopic versus endoscopic transsphenoidal surgeries for Knosp Grades 0-2 nonfunctioning pituitary macroadenomas at a single institution. J Neurosurg. 2014;121(3):511.

45. Thawani JP, Ramayya AG, Pisapia JM, Abdullah KG, Lee JY, Grady MS. Operative strategies to minimize complications following resection of pituitary macroadenomas. J Neurol Surg B Skull Base. 2017;78(2):184-90. https://doi.org/10.1055/s-0036-1597276.

46. Samuel G, Shiley F, Limonadi J, et al. Incidence, etiology, and management of cerebrospinal fluid leaks following trans-sphenoidal surgery. Laryngoscope. 2003;113(8):1283-8. https://doi.org/10.1097/00005537200308000-00003. 
47. Nishioka H, Haraoka J, Ike Da Y. Risk factors of cerebrospinal fluid rhinorrhea following transsphenoidal surgery. Acta Neurochir. 2005;147(11):1163-6. https://doi.org/10.1007/s00701-005-0586-3.

48. Chi F, Wang Y, Lin Y, Ge J, Qiu Y, Guo L. A learning curve of endoscopic transsphenoidal surgery for pituitary adenoma. J Craniofac Surg. 2013;24(6):2064-7. https://doi.org/10.1097/SCS.0b013e3182a24328.

49. Chen XY, Ding CY, You HH, Chen JY, Jiang CZ, Yan XR, et al. Relationship between pituitary adenoma consistency and extent of resection based on tumor/cerebellar peduncle T2-weighted imaging intensity (TCTI) ratio of the point on preoperative magnetic resonance imaging (MRI) corresponding to the residual point on postoperative MRI. Med Sci Monit. 2020;26:e919565. https://doi.org/10.12659/MSM.919565.

50. Azab WA, Nasim K, Abdelnabi EA, Yousef W, Najibullah M, Khan T, et al. Endoscopic endonasal excision of large and giant pituitary adenomas: radiological and intraoperative correlates of the extent of resection. World Neurosurg. 2019;126:e793-802. https://doi.org/10.1016/j.wneu. 2019.02.151.

51. Jakimovski D, Bonci G, Attia M, Shao H, Hofstetter C, Tsiouris AJ, et al. Incidence and significance of intraoperative cerebrospinal fluid leak in endoscopic pituitary surgery using intrathecal fluorescein. World Neurosurg. 2014;82(3-4):e513-23. https://doi.org/10.1016/j.wneu.2013.06.005.

52. Seiler RW, Mariani L. Sellar reconstruction with resorbable vicryl patches, gelatin foam, and fibrin glue in transsphenoidal surgery: a 10-year experience with 376 patients. J Neurosurg. 2000;93(5):762-5. https://doi.org/10. 3171/jns.2000.93.5.0762.

53. Strickland BA, Lucas J, Harris B, Kulubya E, Bakhsheshian J, Liu C, et al. Identification and repair of intraoperative cerebrospinal fluid leaks in endonasal transsphenoidal pituitary surgery: surgical experience in a series of 1002 patients. J Neurosurg. 2018;129(2):425-9. https://doi.org/ 10.3171/2017.4.JNS162451.

54. Mishra SK, Mathew GA, Paul RR, Asif SK, John M, Varghese AM, et al. Endoscopic repair of CSF rhinorrhea: an institutional experience. Iran J Otorhinolaryngol. 2016;28(84):39-43.

55. Halvorsen H, Ramm-Pettersen J, Josefsen R, Rønning P, Reinlie S, Meling $T$, et al. Surgical complications after transsphenoidal microscopic and endoscopic surgery for pituitary adenoma: a consecutive series of 506 procedures. Acta Neurochir. 2014;156(3):441-9. https://doi.org/10.1007/ s00701-013-1959-7.

56. Li A, Liu W, Cao P, Zheng Y, Bu Z, Zhou T. Endoscopic versus microscopic transsphenoidal surgery in the treatment of pituitary adenoma: a systematic review and meta-analysis. World Neurosurg. 2017;101:236-46. https://doi.org/10.1016/j.wneu.2017.01.022.

57. Robins J, Alavi SA, Tyagi AK, Nix PA, Wilson TM, Phillips NI. The learning curve for endoscopic trans-sphenoidal resection of pituitary macroadenomas. A single institution experience, Leeds, UK. Acta Neurochir. 2018;160(1):39-47. https://doi.org/10.1007/s00701-017-3355-1.

58. Lobatto DJ, de Vries F, Zamanipoor NA, Pereira AM, Peul WC, Vliet VT, et al. Preoperative risk factors for postoperative complications in endoscopic pituitary surgery: a systematic review. Pituitary. 2018;21(1):84-97. https:// doi.org/10.1007/s11102-017-0839-1.

59. Boling CC, Karnezis TT, Baker AB, Lawrence LA, Soler ZM, Vandergrift WR, et al. Multi-institutional study of risk factors for perioperative morbidity following transnasal endoscopic pituitary adenoma surgery. Int Forum Allergy Rhinol. 2016;6(1):101-7. https://doi.org/10.1002/alr.21622.

60. Dlouhy BJ, Madhavan K, Clinger JD, Reddy A, Dawson JD, O'Brien EK, et al. Elevated body mass index and risk of postoperative CSF leak following transsphenoidal surgery. J Neurosurg. 2012;116(6):1311-7. https://doi. org/10.3171/2012.2.JNS111837.

61. Bower JE, Bak K, Berger A, Breitbart W, Escalante CP, Ganz PA, et al. Screening, assessment, and management of fatigue in adult survivors of cancer: an American Society of Clinical oncology clinical practice guideline adaptation. J Clin Oncol. 2014;32(17):1840-50. https://doi.org/10.1200/ JCO.2013.53.4495.

\section{Publisher's Note}

Springer Nature remains neutral with regard to jurisdictional claims in published maps and institutional affiliations.
Ready to submit your research? Choose BMC and benefit from:

- fast, convenient online submission

- thorough peer review by experienced researchers in your field

- rapid publication on acceptance

- support for research data, including large and complex data types

- gold Open Access which fosters wider collaboration and increased citations

- maximum visibility for your research: over $100 \mathrm{M}$ website views per year

At BMC, research is always in progress.

Learn more biomedcentral.com/submissions 